\title{
Estudo das metodologias de destilação na quantificação do Nitrogênio das Bases Voláteis Totais em pescada, tilápia e camarão
}

\author{
Study of distillation methodologies for the quantification of nitrogen in \\ the total volatile bases of hake, tilapia and shrimp
}

\section{Autores | Authors}

Laís Henrique CICERO

Universidade Santa Cecilia (UNISANTA) Curso de Ciências Biológicas Santos/SP - Brasil

e-mail: lais_cicero@hotmail.com

\section{Erika Fabiane FURLAN} Rubia Yuri TOMITA

Instituto de Pesca Unidade Laboratorial de Referência em Tecnologia do Pescado Santos/SP - Brasil e-mail: effurlan@pesca.sp.gov.br tomita@pesca.sp.gov.br

\section{Rita de Cassia Boccuzzi PRISCO} Vera Lúcia Tedeschi SAVOY

Instituto Biológico Laboratório de Química Ambiental São Paulo/SP - Brasil e-mail: rprisco@biologico.sp.gov.br savoy@biologico.sp.gov.br

\section{$\triangle$ Cristiane Rodrigues Pinheiro NEIVA}

Instituto de Pesca Unidade Laboratorial de Referência em Tecnologia do Pescado Av. Bartolomeu de Gusmão, 192 CEP: 11030-906 Santos/SP - Brasil e-mail: crpneiva@pesca.sp.gov.br

$\square$ Autor Correspondente / Corresponding Author

Recebido: Nov. 27, 2013

Aprovado: Ago. 18, 2014

\section{Resumo}

O pescado apresenta grande importância no panorama atual e a manutenção da sua qualidade e inocuidade é imprescindível para se obter alimento seguro. Por ser um alimento altamente perecível, o pescado necessita de cuidados em todas as etapas da cadeia produtiva. Um dos métodos utilizados mundialmente para avaliar a qualidade do pescado é a determinação do Nitrogênio das Bases Voláteis Totais (N-BVT), que consiste na quantificação de compostos de baixo peso molecular, como a trimetilamina, dimetilamina e amônia, que são formados durante o processo de deterioração do pescado. O objetivo deste trabalho foi avaliar a eficiência de duas metodologias de quantificação de N-BVT (BRASIL 1981 e BRASIL 1999) por destilação, para três espécies de pescado de importância comercial, assim como buscar a diminuição do volume de amostras e reagentes utilizados. Para verificar a eficiência das metodologias utilizou-se um índice de recuperação, adicionando-se quantidades conhecidas de sulfato de amônio $\left[\left(\mathrm{NH}_{4}\right)_{2} \mathrm{SO}_{4}\right]$ nas amostras e comparando-se o teor de N-BVT recuperado com uma amostra controle. Foram testadas modificações na metodologia BRASIL 1999 quanto à redução nas quantidades de amostra e reagente. Considerando-se o desempenho quanto à recuperação do nitrogênio adicionado às amostras, os resultados demostraram que não houve diferença quanto à recuperação de nitrogênio nos dois métodos (BRASIL 1981 e BRASIL 1999) para as três espécies estudadas, evidenciando-se que ambos podem ser empregados com a mesma eficiência. Os resultados indicam que a redução na quantidade de amostra e reagentes, nas determinações de N-BVT para o método BRASIL 1999, pode interferir significativamente nos resultados.

Palavras-chave: Recuperação; Nitrogênio das Bases Voláteis Totais; Qualidade do pescado.

\section{Summary}

Fish is very important on the current scenario, and the maintenance of its quality and safety is essential to obtain a safe food. Since fish is a highly perishable food, it needs proper care at all stages of its production chain. One of the parameters most widely used worldwide to evaluate fish quality is the determination of the nitrogen of the total volatile bases (TVB-N), which consists of quantifying the low molecular weight compounds such as trimethylamine, dimethylamine and ammonia, formed during deterioration of the fish. The objective of this study was to evaluate the efficiency of two quantification methods of TVB-N by distillation, using three fish species of commercial importance, as well as to search for a more sustainable method using smaller amounts of sample and reagents. The recovery rate was used to verify the efficiency of the methodologies, adding known amounts of ammonium sulphate $\left(\left(\mathrm{NH}_{4}\right)_{2} \mathrm{SO}_{4}\right)$ to samples and comparing the TVB-N recovered from a control sample). In order to reduce the amounts of sample and reagents, modifications of the BRAZIL (1999) methodology were tested. Considering the recovery of the nitrogen added to the samples, the results showed there was no difference in the recovery of nitrogen using the two methods (BRAZIL 1981 and 1999) for the three species, indicating that both could be used with the same efficiency. The results indicated that the reduction in the amounts of sample and reagents used in the TVB-N determination by the BRAZIL (1999) method, could significantly interfere with the results. 


\section{Introdução}

O comércio mundial de produtos pesqueiros alcançou um recorde mundial de 160 milhões de toneladas no ano de 2013, um consumo mundial de $20 \mathrm{~kg}$ de pescado por pessoa/ano, sendo que a aquicultura contribuiu com quase metade desse valor (FAO, 2014). Dentre as espécies de pescado de importância econômica no Brasil podemos destacar as pescadas, a tilápia e o camarão de cultivo (BRASIL, 2011).

O pescado é um alimento que se destaca nutricionalmente, sendo indicado para dietas balanceadas e saudáveis (RUXTON, 2011), mas a após a sua captura ou despesca o produto sofre uma série de alterações físicas, químicas, bioquímicas e microbiológicas. Essas alterações são iniciadas pela ação de enzimas endógenas nos músculos, que hidrolisam gorduras e proteínas (TAVARES e GONÇALVES, 2011).

Além disso, ocorre a degradação de compostos nitrogenados por bactérias, formando principalmente trimetilamina (TMA) e amônia, incrementando o pH ao final da fase de rigor mortis (CLUCAS e SUTCLIFFE, 1988; CONNELL, 1988).

A mensuração da quantidade de Nitrogênio das Bases Voláteis Totais (N-BVT) em uma amostra de músculo de pescado, que indica seu estado de frescor, consiste na quantificação de aminas como TMA, dimetilamina (DMA) e amônia, que são formadas durante o processo de deterioração do pescado (PALMA, 1994; HOWGATE, 2010a).

A quantificação do N-BVT é um dos métodos mais utilizados para a determinação da qualidade do pescado, pois apresenta procedimento analítico simples, de baixo custo e independe de grande aparato de equipamentos (BAIXAS NOGUEIRAS et al., 2001; MALLE e POUMEYROL, 1989; HOWGATE, 2009, 2010a).

De acordo com a Diretiva Europeia 95/149/CEE, o N-BVT deve ser usado quando os métodos sensoriais deixarem dúvidas quanto ao frescor do pescado. Alguns países, como Alemanha, Brasil, Japão, Austrália e Argentina, também adotaram esse teste como parâmetro normatizado de qualidade para pescado (SCHERER et al., 2004).

Trabalhos e documentos sobre a qualidade dos produtos de pescado sugerem limites para N-BVT e N-TMA para consumo humano (CONNELL, 1995; COMISSÃO EUROPEIA, 1995; CODEX ALIMENTARIUS, 2007), com valores diferenciados por grupos de peixes, de acordo com suas características, especialmente quanto à composição química, variando de $25 \mathrm{mg} \mathrm{N-BVT/100} \mathrm{g} \mathrm{em}$ peixes gordos e de carne avermelhada até o limite de 35 mg N-BVT/100 g para espécies magras e de carne branca (HOWGATE, 2010b). A quantidade máxima de N-BVT permitida no Brasil, segundo a Secretaria de Defesa
Agropecuária do Ministério da Agricultura, Pecuária e Abastecimento, é de $30 \mathrm{mg} \mathrm{N} / 100 \mathrm{~g}$ de pescado para as diferentes espécies de peixes, com exceção dos Elasmobrânquios, como os cações, onde a mensuração desses compostos não serve como parâmetro de qualidade (BRASIL, 1997).

A destilação de extratos obtidos a partir dos músculos do peixe, sob pressão reduzida, e o método de microdifusão (célula de Conway) são os métodos mais aceitos e utilizados na atualidade para quantificar o N-BVT (HOWGATE, 2010a). Algumas tecnologias têm sido desenvolvidas para a quantificação da N-TMA, N-DMA e amônia, como cromatografia gasosa e eletroforese capilar, no entanto esses métodos são complexos, demorados e de alto custo, resultando na maior utilização dos métodos tradicionais (HOWGATE, 2010a).

Este trabalho teve como objetivo avaliar duas metodologias de quantificação do N-BVT por destilação citadas na legislação brasileira em três espécies de pescado, considerando o desempenho quanto à recuperação do nitrogênio adicionado à amostra e a possibilidade de diminuição dos volumes de amostra e reagentes utilizados.

\section{Material e métodos}

As análises foram conduzidas na Unidade Laboratorial de Referência em Tecnologia do Pescado do Instituto de Pesca em Santos, SP. Foram utilizadas amostras de três diferentes espécies de pescado, a saber: a pescada goete (Cinoscyon jamaicensis) oriunda da pesca marinha; a tilápia (Oreochromis niloticus) cultivada em água-doce; e o camarão de cultivo (Litupenaeus vanamei).

Para obtenção de amostras homogêneas e utilização do mesmo lote de amostra em todos os ensaios, lotes de pescada e tilápia foram descabeçados e eviscerados e, então, processados com a tecnologia da Carne Mecanicamente Separada - CMS, conforme Neiva e Gonçalves (2011), com equipamento tipo tambor perfurado, o qual separa a carne dos ossos sem propiciar trituração da carne, pele e ossos e sem alterar a constituição do músculo original.

A amostra de camarão, após descabeçamento e descasque manual dos espécimes, foi triturada em equipamento tipo "cutter". As três amostras foram rapidamente congeladas em ultrafreezer $\mathrm{a}-80^{\circ} \mathrm{C}$ visando manter sua estabilidade e descongeladas em temperatura de refrigeração (entre $4{ }^{\circ} \mathrm{C}$ e $7{ }^{\circ} \mathrm{C}$ ) para a realização das análises.

As metodologias de quantificação de N-BVT (mg $\mathrm{N} / 100 \mathrm{~g}$ de pescado) utilizadas foram as de destilação citadas na legislação brasileira (BRASIL, 1981, 1999). Os dois métodos utilizam uma solução de ácido 
tricloroacético - TCA para a extração do nitrogênio proteico da amostra, que após filtração resulta em um extrato límpido contendo o nitrogênio volátil. No entanto, as referidas metodologias utilizam diferentes agentes alcalinizantes antes da destilação por arraste de vapor, a metodologia BRASIL 1981 utiliza hidróxido de sódio - NaOH, enquanto a BRASIL 1999, o óxido de magnésio - MgO

Para os testes de comparação das referidas metodologias de destilação foram utilizadas as três amostras de pescado anteriormente citadas, pescada, tilápia e camarão. Todas as amostras foram fortificadas com $5 \mathrm{~mL}$ de solução de sulfato de amônio (1\%), equivalente a 10,6 mg de nitrogênio (N) em cada alíquota de amostra. Para chegar ao valor da porcentagem ou índice de recuperação (\%R) do nitrogênio adicionado foi realizado o cálculo:

$$
\% R=(\text { Nf-Nc }) \times 100 / \mathbf{1 0 , 6}
$$

em que

\%R: Porcentagem ou índice de recuperação;

Nf: mg N-BVT/100 g da amostra fortificada [com adição de $\left(\mathrm{NH}_{4}\right)_{2} \mathrm{SO}_{4}$ ];

Nc: Média de N-BVT (100 g) na amostra controle [sem adição de $\left(\mathrm{NH}_{4}\right)_{2} \mathrm{SO}_{4}$ ];

10,6: $\mathrm{mg} \mathrm{N}\left(\mathrm{NH}_{4}\right)_{2} \mathrm{SO}_{4}$ adicionado para fortificação.

Buscando-se avaliar as possíveis modificações quanto à diminuição das quantidades de amostra e reagentes, utilizou-se a amostra de pescada e a metodologia BRASIL 1999, a qual apresentou menor nível de variação nos resultados. Foram realizados três ensaios com sete replicatas ou repetições de cada tratamento. Testou-se o método original (Tratamento A), que utiliza $100 \mathrm{~g}$ de amostra e $300 \mathrm{~mL}$ de TCA, e outras duas alterações do mesmo método utilizando-se: $50 \mathrm{~g}$ de amostra e $150 \mathrm{~mL}$ de TCA (Tratamento B) e $10 \mathrm{~g}$ de amostra e $50 \mathrm{~mL}$ de TCA (Tratamento C).

Os dados obtidos pelos distintos métodos de determinação do N-BVT foram analisados pelo pacote estatístico STATA 8.0 (StatSoft Inc., USA) e expressos como média e desvio padrão. Comparações longitudinais foram conduzidas utilizando-se ANOVA one-way e as diferenças significativas entre os diferentes métodos estudados $(p<0,01)$ foram estabelecidas através do teste de Dunnett, o qual realiza múltiplas comparações onde apenas um tratamento serve de referência, ou seja, quando se tem tratamento ou grupo controle.

\section{Resultados e discussão}

\subsection{Avaliações dos métodos de destilação}

A literatura científica cita a utilização de algumas substâncias, como o sulfato de amônio $\left[\left(\mathrm{NH}_{4}\right)_{2} \mathrm{SO}_{4}\right]$ e o cloreto de amônio $\left[\left(\mathrm{NH}_{4}\right) \mathrm{Cl}\right.$, atuando como fortificadoras, ou seja, com o papel de incrementar o nitrogênio não proteico em ensaios de quantificação de N-BVT (AMANAJÁS, 1985; ANTONACOPOULOS e VYNCKE, 1989, HOWGATE, 2009, 2010a).

$A$ adição de $\left(\mathrm{NH}_{4}\right)_{2} \mathrm{SO}_{4}$ às amostras também foi referendada no trabalho de Villarreal (2007), demonstrando a estabilidade desse composto e um comportamento similar ao nitrogênio não proteico.

Entretanto, testes preliminares conduzidos utilizando-se o $\left(\mathrm{NH}_{4}\right)_{2} \mathrm{SO}_{4}$ e o $\left(\mathrm{NH}_{4}\right) \mathrm{Cl}$, demonstraram que $\mathrm{o}\left(\mathrm{NH}_{4}\right)_{2} \mathrm{SO}_{4}$ apresentava melhor comportamento quanto à recuperação do nitrogênio - $\mathrm{N}$ quando utilizado na concentração de $1 \%$.

Os resultados médios da recuperação de N-BVT para as diferentes matrizes foram comparados com os das amostras controle [sem a adição de $\left(\mathrm{NH}_{4}\right)_{2} \mathrm{SO}_{4}$ ]. Na Tabela 1 estão descritos os resultados médios de N-BVT encontrados nas amostras controle de cada espécie a partir das metodologias normatizadas.

Como visualizado na Tabela 1, as três amostras estudadas apresentaram valores de N-BVT abaixo do limite estipulado pelo Ministério da Agricultura, Pecuária e Abastecimento (BRASIL, 1997), de $30 \mathrm{mg} \mathrm{N} / 100 \mathrm{~g}$ de pescado, em ambos os métodos.

A análise estatística indicou que os valores obtidos de N-BVT pelos dois métodos (BRASIL, 1981, 1999)), para uma mesma matriz, variaram significativamente $(p<0,01)$. Essa variação entre métodos se deu para todas as matrizes (camarão, tilápia e pescada), demonstrando que os métodos mencionados na legislação brasileira resultam em valores de N-BVT significativamente distintos, evidenciando-se a necessidade de estudos sobre a performance das metodologias para distintas matrizes. O teste de Dunnett indicou que o método BRASIL 1999 sempre quantificou valores de N-BVT significativamente inferiores aos do método BRASIL 1981. Na Tabela 2 estão descritos os resultados obtidos no teste de recuperação do nitrogênio utilizando-se as diferentes amostras fortificadas com solução a $1 \%$ de $\left(\mathrm{NH}_{4}\right)_{2} \mathrm{SO}_{4}$ analisadas pelas duas metodologias.

Antonacopoulos e Vyncke (1989), trabalhando com diferentes espécies de peixe, descreveram que o método que usa o óxido de magnésio ( $\mathrm{MgO}$ ) como agente alcalinizante, como é o caso do método BRASIL 1981,

Tabela 1. Valores médios de N-BVT (mg N/ 100 g) determinados nas amostras controle de pescada, tilápia e camarão utilizando-se os métodos BRASIL 1981 e BRASIL 1999.

\begin{tabular}{lcc}
\hline Espécie & BRASIL 1981 & BRASIL 1999 \\
\hline Pescada & $18,34^{\mathrm{a}} \pm 0,9$ & $11,35^{\mathrm{b}} \pm 0,5$ \\
Tilápia & $15,19^{\mathrm{a}} \pm 1,09$ & $9,56^{\mathrm{b}} \pm 1,19$ \\
Camarão & $28,85^{\mathrm{a}} \pm 0,60$ & $12,95^{\mathrm{b}} \pm 1,2$ \\
\hline
\end{tabular}

Letras sobrescritas iguais na mesma linha indicam igualdade estatística; média $(n=3) \pm$ desvio padrão. 
Estudo das metodologias de destilação na quantificação do Nitrogênio das Bases Voláteis Totais em pescada, tilápia e camarão CICERO, L. H. et al.

Tabela 2. Nitrogênio total (NT), Nitrogênio recuperado (Nrec), ambos expressos em mg N/100 g de amostra e Porcentagem ou Índice de Recuperação de N-BVT (R\%), segundo cálculo citado, em amostras fortificadas com $\left(\mathrm{NH}_{4}\right)_{2} \mathrm{SO}_{4}$, em três espécies de pescado, utilizando os métodos de destilação BRASIL 1981 e BRASIL 1999.

\begin{tabular}{|c|c|c|c|c|c|c|}
\hline \multirow{2}{*}{ Espécie } & \multicolumn{3}{|c|}{ BRASIL 1981} & \multicolumn{3}{|c|}{ BRASIL 1999} \\
\hline & NT (mg N/100 g) & Nrec (mg N/100 g) & $\mathbf{R} \%$ & NT (mg N/100 g) & Nrec (mg N/100 g) & $\mathbf{R} \%$ \\
\hline Pescada & 29,71 & $11,36^{a} \pm 1,54$ & 107,2 & 22,53 & $11,18^{a} \pm 1,40$ & 105,5 \\
\hline Tilápia & 24,84 & $9,65^{a} \pm 1,49$ & 91,0 & 20,48 & $10,92^{a} \pm 1,07$ & 103,0 \\
\hline Camarão & 38,86 & $10,01^{a} \pm 1,03$ & 94,4 & 22,04 & $9,09^{a} \pm 0,67$ & 85,7 \\
\hline
\end{tabular}

Letras sobrescritas iguais na mesma linha indicam igualdade estatística; média $(n=3) \pm$ desvio padrão.

Tabela 3. Teor de N-BVT (mg N/100 g) em pescada utilizando-se metodologia descrita em BRASIL 1999 - Tratamento A e modificações nessa metodologia - Tratamentos B e C em três repetições de ensaio.

\begin{tabular}{cccc} 
Ensaio & \multicolumn{3}{c}{ Tratamentos } \\
& \multicolumn{1}{c}{$\mathbf{A}$} & \multicolumn{1}{c}{$\mathbf{B}$} & C \\
\hline $\mathbf{1}$ & $8,96^{\mathrm{a}} \pm 0,49$ & $8,25^{\mathrm{b}} \pm 0,38$ & $7,62^{\mathrm{c}} \pm 0,84$ \\
$\mathbf{2}$ & $8,96^{\mathrm{a}} \pm 0,49$ & $8,01^{\mathrm{b}} \pm 0,29$ & $7,75^{\mathrm{c}} \pm 0,76$ \\
$\mathbf{3}$ & $9,36^{\mathrm{a}} \pm 0,59$ & $8,75^{\mathrm{b}} \pm 1,2$ & $6,9^{\mathrm{c}} \pm 0,76$ \\
\hline
\end{tabular}

Letras sobrescritas iguais na mesma linha indicam igualdade estatística; $\mathrm{A}=$ método original, $\mathrm{B}=50 \mathrm{~g}$ de amostra $+150 \mathrm{~mL}$ de TCA $5 \%, C=10 \mathrm{~g}$ de amostra $+50 \mathrm{~mL}$ de TCA $5 \% ; n=7 ; p<0,01$, em três ensaios.

propicia melhor recuperação que aquele que utiliza o hidróxido de sódio $(\mathrm{NaOH})$, fato que ocorreu no presente estudo para as amostras de pescada e camarão.

Apesar de os resultados aparentemente parecerem diferentes, a análise estatística do teste de recuperação indicou que eles não variaram significativamente $(p<0,01)$ entre os métodos para as diferentes matrizes de pescado estudadas, indicando que ambas as metodologias podem ser empregadas com a mesma eficiência.

\subsection{Modificações na metodologia de destilação - BRASIL 1999}

A metodologia de destilação BRASIL 1999 foi selecionada para testar algumas modificações empregando-se a amostra de peixe magro com carne branca (pescada), visando a redução nas quantidades de amostra e, especialmente, no volume de TCA utilizado na extração, sendo essa uma prática mundialmente comum em laboratórios de análise de pescado. Assim, fez-se um controle aqui denominado Tratamento A, no qual utilizou-se a quantidade de amostra e reagente originalmente descritos no método (100 gramas em $300 \mathrm{~mL}$ de TCA 5\%), e esse foi comparado ao Tratamento $B$, que utilizou metade do método original (50 gramas em $150 \mathrm{~mL}$ de TCA 5\%), e ao Tratamento C, que utilizou uma proporção diferente das demais (10 gramas em 50 $\mathrm{mL}$ de TCA 5\%).

Na Tabela 3 são apresentados os resultados obtidos nos ensaios com a metodologia de destilação descrita em BRASIL 1999 e com as modificações propostas.
Os valores de N-BVT obtidos nos três tratamentos, nas três repetições de ensaio (ensaios 1, 2 e 3), variaram significativamente entre si $(p<0,01)$, sendo que os valores obtidos pelos tratamentos B e C apresentaram-se sempre significativamente inferiores aos obtidos pelo método A. Ademais os valores obtidos pelo método $C$ foram significativamente inferiores aos obtidos pelo método B quando consideramos a soma das três repetições de ensaio $(n=21)$.

Os resultados indicam que a redução nas quantidades de amostra e reagentes comumente utilizados nas determinações de N-BVT pode interferir significativamente nos resultados obtidos.

No entanto é importante buscar alternativas às metodologias normalmente utilizadas no sentido de se reduzir a utilização de reagentes e a consequente geração de resíduos químicos. Os resultados obtidos indicam a necessidade de se aprofundar esse tipo de estudo, viabilizando modificações que não afetem o resultado final da análise, como aqui demonstrado.

\section{Conclusão}

A avaliação das duas metodologias de quantificação do N-BVT utilizando-se três espécies de pescado considerando o desempenho quanto à recuperação de nitrogênio adicionado evidenciou que não houve diferença quanto ao desempenho dos métodos BRASIL 1981 e BRASIL 1999 para as três matrizes estudadas e que ambas metodologias podem ser empregadas com a mesma eficiência. Os resultados indicam que a redução nas quantidades de amostra e reagentes utilizadas na determinação de N-BVT no método BRASIL 1999 pode interferir significativamente nos resultados.

\section{Agradecimentos}

Ao CNPq, pelo suporte financeiro (Edital $n$. 064/2008) e pela bolsa de iniciação científica - PIBIC da aluna Laís Henrique Cicero.

\section{Referências}

AMANAJÁS, C. C. Determinação dos Compostos Básicos Totais do Pescado e o seu Potencial para Avaliação do Frescor. 1985. 110 f. Dissertação (Mestrado em Tecnologia de 
Estudo das metodologias de destilação na quantificação do Nitrogênio das Bases Voláteis Totais em pescada, tilápia e camarão CICERO, L. H. et al.

Alimentos)-Universidade Estadual de Campinas, Campinas, 1985.

ANTONACOPOULOS, N.; VYNCKE, W. Determination of Volatile Basic Nitrogen in Fish: a Third Collaborative Study by the West European Fish Technologists' Association (WEFTA). Zeitschrift für Lebensmittel-Untersuchung und Forschung, Berlin, v. 189, n. 4, p. 309-316, 1989. http://dx.doi.org/10.1007/BF01683206

BAIXAS NOGUEIRAS, S.; BOVER-CID, M.; VIDAL-CAROU, M. C.; VECIANA-NOGUÉS, M. T.; MARINÉ-FON, A. Trimethylamine and Total Volatile Basic Determination by Flow Injection/ Gas Diffusion in Mediterranean hake (Merluccius merluccius). Journal of Agricultural and Food Chemistry, California, v. 49, n. 4, p. 1681-1686, 2001. http://dx.doi.org/10.1021/jf000649n

BRASIL. Ministério da Agricultura, Pecuária e Abastecimento. Secretaria Nacional de Defesa Agropecuária. Portaria n 01 , de 07 de Outubro de 1981. Aprova os Métodos Analíticos para Controle de Produtos de Origem Animal e seus Ingredientes, Constituindo-se em Métodos Microbiológicos e Métodos Físicos e Químicos. Diário Oficial da União, Poder Executivo, Brasília, DF, 13 out. 1981. Seção 1. Disponível em: <http://sistemasweb. agricultura.gov. br/sislegis/action/detalhaAto. do?method=consu ItarLegislacaoFederal>. Acesso em: 22 nov. 2013.

BRASIL. Ministério da Agricultura, Pecuária e Abastecimento. Secretaria de Defesa Agropecuária. Portaria n 185, de 13 de Maio de 1997. Aprova o Regulamento Técnico de Identidade e Qualidade de Peixe Fresco. Diário Oficial da União, Poder Executivo, Brasília, DF, 19 Maio 1997. Disponível em:<http://www.cda.sp.gov.br/www/legislacoes/popup. php?action=view\&idleg=670 > . Acesso em: 22 nov. 2013.

BRASIL. Ministério da Agricultura, Pecuária e Abastecimento. Secretaria de Defesa Agropecuária. Instrução Normativa $n^{\circ}$ 20, de 21 de Julho de 1999. Métodos analíticos físico químicos para controle de produtos cárneos e seus ingredientes - sal e salmoura. Diário Oficial da União, Poder Executivo, Brasília, DF, 27 Jul. 1999. Seção 1. Disponível em: <http://sistemasweb. agricultura.gov.br/sislegis/action/detalhaAto. do?method=grav arAtoPDF\&tipo $=$ INM \&numeroAto $=20 \&$ seqAto $=000 \&$ valorAno $=$ $1999 \&$ orgao $=S D A \&$ codTipo $=\&$ des $\mid$ tem $=\&$ seqNota $=>$. Acesso em: 22 nov. 2013.

BRASIL. Ministério da Pesca e Aquicultura. Boletim Estatístico da Pesca e Aquicultura. Brasília: Ministério da Pesca e Aquicultura, 2011. Disponível em: <http://www.mpa.gov. br/index.php/imprensa/noticias/2140-mpa-lanca-boletim-

estatistico-da-pesca-e-aquicultura-2011 >. Acesso em: 22 maio 2014.

CLUCAS, I. J.; SUTCLIFFE, P. J. Introducción al Manejo y Procesamiento de Productos del Pescado. Chatham: Instituto de Desarrollo de Recursos, 1988. 144 p.

CODEX ALIMENTARIUS. Code of Practice for Fish and Fishery Products: CAC/RCP52-2003. Rome: Codex Committee on Fish and Fishery Products, 2007. Disponível em: <www. codexalimentarius.net/download/standards/10273/CXP_052e. pdf>. Acesso em: 24 abr. 2008.
CONNELL, J. J. Control de la Calidad del Pescado. Zaragoza: Acribia. 1988. $236 \mathrm{p}$.

CONNELL, J. J. Control of Fish Quality. 4th ed. Farnham: Fishing News, 1995. 256 p.

COMISSÃO EUROPEIA. Diretiva Europeia 95/149/CE. Decisão da Comissão, de 8 de Março de 1995, que Fixa os Valores de Azoto Basico Volátil Total (ABVT) para Determinadas Categoriuas de Produtos da Pesca e os Métodos de Análise a Utilizar. Official Journal of the European Union, European Union, 29 abr. 1995. $n^{\circ}$ L 097, p. 0084-0087.

HOWGATE, P. Traditional Methods. In: REHBEIN, H.; OEHLENSCHLÄGER, J. (Ed.) Fishery Products: Quality, Safety and Authenticity. Oxford: Wiley-Blackwell, 2009. p. 19-41. cap. 2.

HOWGATE, P. A Critical Review of Total Volatile Bases and Trimethylamine as Indices of Freshness of Fish. Part 1: Determination. Electronic Journal of Environmental, Agricultural and Food Chemistry, West Sussex, v. 9, n. 1, p. 29-57, 2010a.

HOWGATE, P. A Critical Review of Total Volatile Bases and Trimethylamine as Indices of Freshness of Fish. Part 2: Formation of the Bases, and Application in Quality Assurance. Electronic Journal of Environmental, Agricultural and Food Chemistry, West Sussex, v. 9, n. 1, p. 58-88, 2010b.

MALLE, P.; POUMEYROL, M. A New Chemical Criterion for the Quality Control of Fish: Trimethylamine/Total Volatile Basic Nitrogen (\%). Journal of Food Protection, Des Moines, v. 52 , n. 6, p. 419-423, 1989.

NEIVA, C. R. P.; GONÇALVES, A. A. Tecnologia de Carne Mecanicamente Separada de Pescado. In: GONÇALVES, A. A. (Ed.). Tecnologia do Pescado. São Paulo: Atheneu, 2011. p. 197-208. cap. 2.1.8.

ORGANIZAÇÃO DAS NAÇÕES UNIDAS PARA AGRICULTURA E ALIMENTAÇÃO - FAO. Comércio Global de Peixes Atinge Níveis Recordes. Brasília. Disponível em: <https://www.fao.org. br/cgpanr.asp>. Acesso em: 7 mar. 2014.

PALMA, E. J. Frescura del Pescado y Mecanismos. In: CURSO INTERNACIONAL TECNOLOGIA DE PROCESAMIENTO DE PRODUCTOS PESQUEROS "INFORMACIÓN BASICA: QUIMICA, BIOQUIMICA E MICROBIOLOGIA", 10., 1994, Peru. Peru: Instituto Tecnologico Pesquero Del Peru, 1994. p. 59-73.

RUXTON, C. H. S. The Benefits of Fish Consumption. Nutrition Bulletin, London, v. 36, n. 1, p. 6-19, 2011. http://dx.doi. org/10.1111/j.1467-3010.2010.01869.x

SCHERER, R.; DANIEL, A. P.; AUGUSTI, P. R.; LAZZARI, R.; LIMA, R. L.; FRIES, L. L. M.; RADUNZ NETO, J.; EMANUELLI, J. Efeito do Gelo Clorado sobre Parâmetros Químicos e Microbiológicos da Carne de Carpa Capim (Ctenopharyngodon idella). Ciências e Tecnologia de Alimentos, Campinas, v. 24, n. 4 , p. 680-684, 2004. Disponível em: <http://www.scielo.br/scielo. 
Estudo das metodologias de destilação na quantificação do Nitrogênio das Bases Voláteis Totais em pescada, tilápia e camarão CICERO, L. H. et al.

php?script=sci_arttext\&pid=S0101-20612004000400034\&lng= en\&nrm=iso>. Acesso em: 20 maio 2013.

TAVARES, M.; GONÇALVES, A. A. Aspectos Físico-químicos do Pescado. In: GONÇALVES, A. A. (Ed.). Tecnologia do Pescado. São Paulo: Atheneu, 2011. p. 10-20. cap. 1.2.
VILLARREAL, B. P. Methods for the Sensory Evaluation of the Quality of Fresh Fish. Bizkaia: AZTI Tecnalia, 2007. Disponível em: <http://valid.aztionline.com/index.php?idioma=en\&secci on $=6 \&$ ctipo $=2 \&$ contenido $=338 \&$ menu $=136 \&$ submenu $=217$. Acesso em: 18 jun. 2013. 